Original Articles

\title{
Coronary Atherosclerosis in Youths in Kyushu Island, Japan : Histological Findings and Stenosis
}

\author{
Atsushi Kisanuki, Yujiro Asada, Yuichiro Sato, Kousuke Marutsuka, Kenji Takeda, \\ and Akinobu Sumiyoshi
}

First Department of Pathology, Miyazaki Medical College, Miyazaki, Japan

\begin{abstract}
We histologically examined the coronary arteries of 52 autopsied cases of the youths ( 3 to 39 years of age, mean 28.5 years) in Kyushu island, Japan, without clinical events of coronary artery diseases. The coronary artery specimens were taken from the proximal portions of the right coronary artery (Seg. 1), the left anterior descending artery (Seg. 6), and the macroscopically most stenotic region (ST). Atherosclerotic lesions were histologically classified into four types: concentric fibrous, eccentric fibrous, concentric lipid-rich, or eccentric lipid-rich type. The degrees of stenosis ( $<25 \%, 25-50 \%, 50-75 \%,>75 \%)$ were morphometrically evaluated. The majority of coronary arteries with under $50 \%$ stenosis were of the concentric fibrous type. Lipid-rich types of coronary atherosclerosis increased in the coronary arteries with over 50\% stenosis and were observed in the Seg. 6 and ST, while $\mathbf{7 0} \%$ of Seg. 1 lesions with over $50 \%$ stenosis were of a fibrous type. Serum cholesterol levels of patients with a lipid-rich type of coronary atherosclerosis were significantly higher than those with a fibrous type. These results suggested that the early stage of coronary atherosclerosis in Japanese youths is mainly of a concentric fibrous type, which later develops to a lipid-rich type. Hypercholesterolemia would promote the progression of atherosclerosis. J Atheroscler Thromb, $2000 ; 6: 55-59$.
\end{abstract}

Key words : Japanese youth, Autopsy, Risk factor, Fibrous lesion

\section{Introduction}

Atherosclerosis is a complex, slowly progressive, chronic disease of the arteries that is initiated early in life. The disease may have a long latent period before the development of clinical manifestations. Risk factors for atherosclerosis including cigarette smoking, hypercholesterolemia, diabetes mellitus, and hypertension have been well established (1).

It has generally been considered that the Japanese have less severe atherosclerosis than Caucasians. However, it is obvious that the tendency of dietary habits in Japanese has recently become more westernized and the incidence of ischemic heart disease has gradually in-

Address for correspondence: Atsushi Kisanuki, First Department of Pathology, Miyazaki Medical College, 5200 Kihara, Kiyotake, Miyazaki 889-1692, Japan.

Received August 16, 1999.

Accepted for publication November 24, 1999. creased in Japan (2). Much attention therefore has been paid to the problem of atherosclerosis and prevention of hyperlipidemia in Japanese.

Several studies of coronary atherosclerosis have been reported in Western countries (3-6). A few pathological studies of atherosclerosis of Japanese have been reported $(7,8)$, however, no study of the correlation between histological changes and stenosis of coronary atherosclerosis has been performed. In this study, we histologically and histometrically examined coronary atherosclerosis in Japanese youths (under 40 years of age) together with an analysis of the antemortem risk factors.

\section{Materials and Methods}

\section{Collection of coronary arteries}

We examined 52 autopsied cases who ranged in age from 3 to 39 years (mean age 28.5), which were autopsied between January 1991 and December 1995 in 11 hospitals 
in Kyushu island, Japan. No cases had clinical events of coronary artery diseases. The distribution of sex and age is shown in Table 1. The causes of death of these cases are shown in Table 2. Malignancy, including leukemia and lymphoma, accounted for $58 \%$ of the total cases. Three cases of violent death were included.

Three cross sections of coronary arteries were taken from each heart fixed in 10\% formalin ; the right coronary artery (Seg. 1), left anterior descending branch (Seg.6), and the most stenotic portion which was decided macroscopically in the cross sections at $3-\mathrm{mm}$ intervals. The segments of the most stenotic lesion are shown in Table 3. Bifurcated regions of arterial branches were excluded from the tissue sampling. The samples were embedded in paraffin. Hematoxylin and eosin and elastica van Gieason stains were used.

Table 1. Sex and age distribution of the examined cases

\begin{tabular}{cccc}
\hline age & male & female & Total \\
\hline $0-9$ & 1 & 0 & 1 \\
$10-19$ & 8 & 2 & 10 \\
$20-29$ & 12 & 2 & 14 \\
$30-39$ & 18 & 9 & 27 \\
Total & 39 & 13 & 52 \\
\hline
\end{tabular}

Table 2. The main cause of death in of 52 cases examined

\begin{tabular}{|c|c|c|}
\hline \multirow[t]{3}{*}{ malignancy } & \multicolumn{2}{|l|}{30} \\
\hline & \multirow{3}{*}{$\begin{array}{l}\text { leukemia/lymphoma } \\
\text { sarcoma }\end{array}$} & 9 \\
\hline & & 18 \\
\hline sarcoma & & 3 \\
\hline \multicolumn{2}{|c|}{ circulatory disorders $\quad 6$} & \\
\hline \multicolumn{2}{|c|}{ cerebral attack } & 2 \\
\hline \multicolumn{2}{|c|}{ pulmonary hypertension } & 2 \\
\hline \multicolumn{2}{|c|}{ esophageal varices, ruptured } & 2 \\
\hline autoimmune disease & 2 & \\
\hline \multirow{2}{*}{ pneumonia } & 2 & \\
\hline & 6 & \\
\hline injury, trauma & 3 & \\
\hline \multicolumn{2}{|c|}{ others (pancreatitis, aplastic anemia) } & \\
\hline \multicolumn{2}{|c|}{ unknown } & \\
\hline Total & 52 & \\
\hline
\end{tabular}

Table 3. Segments of the most stenotic lesion

\begin{tabular}{ccc}
\hline Coronary a. & Branch & Number of cases \\
\hline LAD & Seg. 5 & 1 \\
LCX & Seg. 6, Seg. 7 & 20 \\
RCA & Seg. 11 & 1 \\
\hline
\end{tabular}

Coronary a: Coronary artery, LAD : Left anterior descending branch, LCX: Left circumflex branch, RCA : Right coronary artery

\section{Morphological examination of atherosclerotic lesions}

The lesions were histologically classified into four types (Fig. 1) : concentric fibrous, eccentric fibrous, concentric lipid-rich, and eccentric lipid-rich type according to Davies' classification (9). We defined the eccentric type as coronary atherosclerosis in which the thickest intimal portion was over 4 times as thick as the thinnest one. Intimal lesions including a pool of free extracellular lipid were defined as lipid-rich type, and lesions composed predominantly of fibromuscular tissue (smooth muscle cells and extracellular matrix) as the fibrous type (9). Luminal stenosis of each segment was assessed using a computerized image analyzer (MacScope Ver. 1.2, Mitani Co., Fukui, Japan). In brief, the area of the lumen (L1) and the inside area of the internal elastic lumina (L2) were measured, and the percentage of luminal stenosis [(L2L1) $\times 100 /$ L2] was calculated, then divided into four degrees ( $<25 \%, 25-50 \%, 50-75 \%,>75 \%)$.

\section{Risk factors}

We obtained the antemortem clinical data including serum total cholesterol (TC), serum triglyceride (TG), blood pressure, and smoking index (Brinkmann index), from 39 of the 52 autopsied cases $(75 \%)$. The data were evaluated statistically using the unpaired Student's t-test. A $p$ value less than 0.05 was accepted as significant.

\section{Results}

\section{Histopathological findings}

The results are shown in Fig. 2. All the segments of coronary arteries with under $25 \%$ stenosis were of a fibrous type, mainly concentric, and the majority of the segments with $25-50 \%$ stenosis were also of a fibrous type in each segment. The proportion of the lipid-rich lesions increased in the segments showing over 50\% stenosis. All lipid rich lesions in this study were of the eccentric stenotic type. In the lesions with 50-75\% stenosis, a half or more lesions of Seg. 6 and ST were of the eccentric lipid-rich type, whereas the lesions of Seg. 1 were predominantly of a fibrous type even with over$50 \%$ stenosis. Lipid-rich types of atherosclerosis were predominantly observed in the segments with over-75\% stenosis, although the number of such specimens was very small in this study.

\section{Analysis of risk factors}

We examined the correlation between risk factors and histological types (fibrous or lipid-rich type) of the most stenotic lesions. Serum TC levels were significantly higher in patients with lipid-rich types than with fibrous types (Fig. 3), but no significant relationship was observed between serum TG or smoking index and histological type (data not shown). 
concentric fibrous

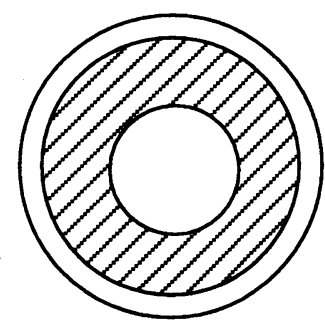

concentric lipid-rich

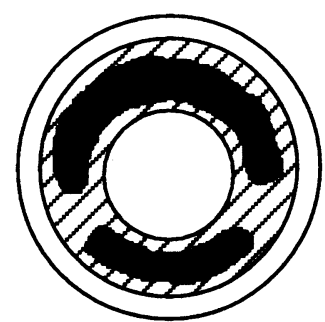

Q7 fibro-muscular tissue eccentric fibrous

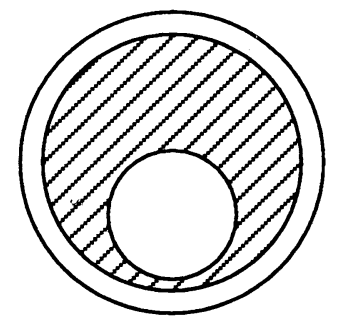

eccentric lipid-rich

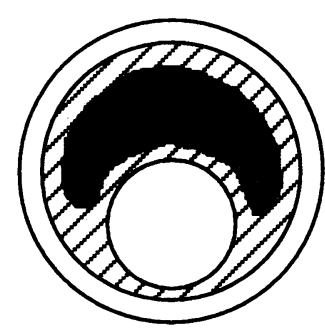

extracellular lipid

Fig. 1. Schema of four type of coronary atherosclerotic lesions.

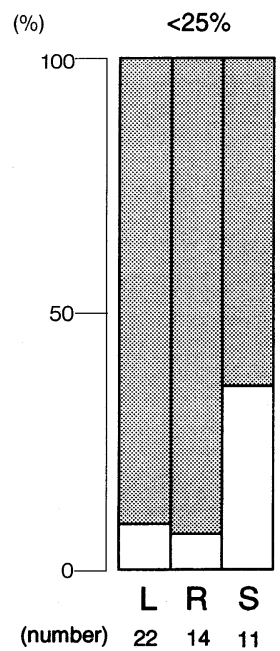

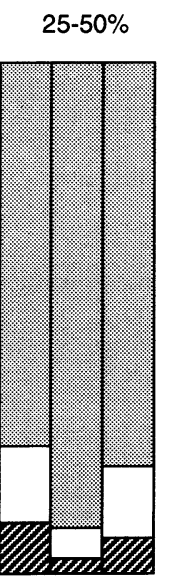

L R S

$20 \quad 34 \quad 28$

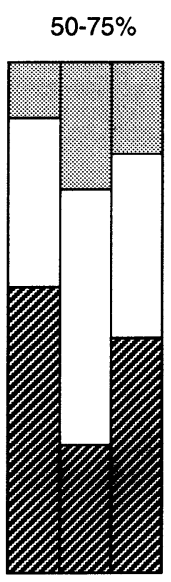

L R S
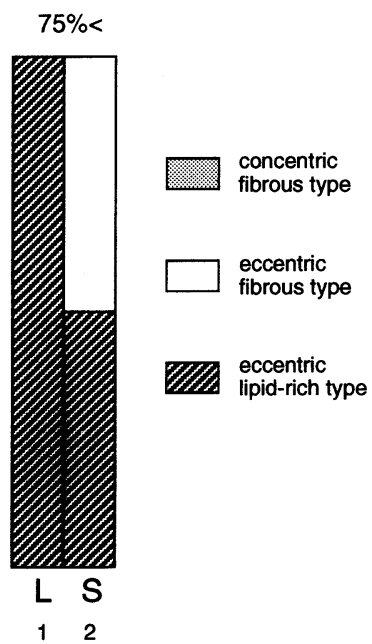

Fig. 2. The relation between coronary stenosis and histological types (52 case, 156 section). L : left coronary artery, anterior descending branch (Seg. 6), R: right coronary artery (Seg. 1), S: the most stenotic region.

\section{Discussion}

The results of the present study showed that, in Japanese youths, the coronary atherosclerosis with under $50 \%$ stenosis was mainly of a concentric fibrous type and, with the progression of atherosclerosis, the lesions would develop to an eccentric lipid-rich type. The development of lipid-rich lesions was considered to be correlated to hypercholesterolemia.

The Pathobiological Determinants of Atherosclerosis in Youth (PDAY) study has clearly demonstrated a strong relation between coronary atherosclerosis and cardiovascular risk factors in young people (10). Hyperlipidemia is well known to be one of the major risk factors for atherosclerosis. In addition, it is generally accepted that atherosclerosis starts from the infiltration of lipoproteins 


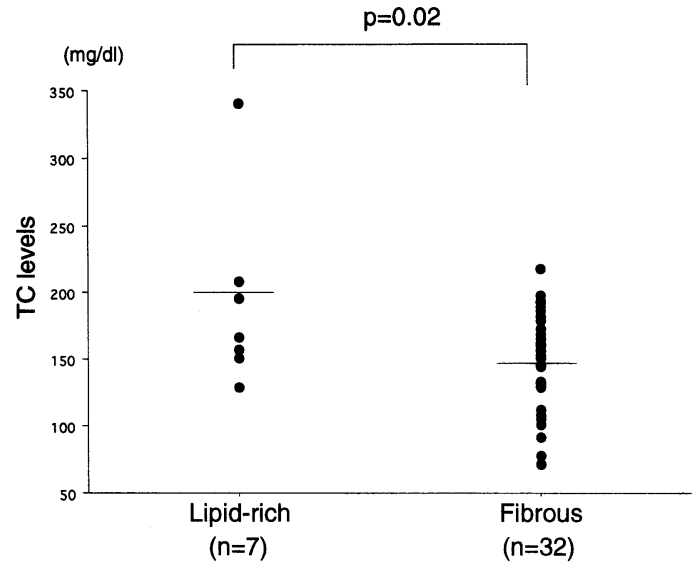

Fig. 3. Correlation between serum cholesterol levels and histological finding of coronary atherosclerosis.

into the arterial walls (11). Such lipoproteins are modified by oxidation, and monocytes subsequently migrate into the intima and are transformed into foam cells (12). It is therefore considered that lipid-rich lesions could be predominant in even in early stage coronary atherosclerosis.

However, our present have showed that the majority of early stage coronary atherosclerosis cases (under 50\% stenosis) are of the fibrous type, and it is in the rather late stage that the lipid-rich type of lesions appears predominantly. In agreement with our results, Kragel et al. (4) reported that fibrous tissue was mainly noted in lesions with under $50 \%$ stenosis, and calcium and pultaceous debris were present in those with over-50\% stenosis. Additionally Davies (9) showed that, even in over $75 \%$ stenotic lesions, fibrous lesions comprised $60 \%$ of coronary atherosclerosis cases. Our present results, together with these previous reports, indicate that proliferation of smooth muscle cells with production of extracellular matrix in the intima is a crucial event in the early stage of coronary atherosclerosis.

On the other hand, some differences between coronary and aortic atherosclerosis have been reported. The PDAY study has shown that atherosclerotic lesions are observed earlier and at a higher prevalence in the aortae than in the coronary arteries, and lipid-rich lesions are observed more frequently in the aortae (10). A few previous studies of Japanese youths $(7,13)$ reported similar results, although the severity of atherosclerosis in Japanese was much lower than that in Americans. The atherosclerotic Index of the aortae was much greater than that of the coronary arteries in each decade of age (14).

It is interesting that a histological difference was observed between the right (Seg. 1) and left (Seg. 6) coronary arteries. The right coronary arteries showed a more predominantly fibrous type than the left even in the over$50 \%$-stenotic lesions. The right and left coronary arteries undergo different ranges of motion during each cardiac cycle, the right exhibiting greater angulation, tortuosity and lateral motion (15). The differences of these anatomical and physiological aspects may affect the histological difference between the sides.

The serum TC levels were significantly higher in patients with lipid rich type lesions than in those with fibrous type. It is well known that hyperlipidemia plays a critical role in the progression of atherosclerosis, especially in developing a large lipid core. Our results support the previous studies $(7,16)$. While smoking index did not correlate with the histological difference of atherosclerotic lesions in this study. It has been reported that atherosclerotic lesions of the coronary arteries and aortae were more frequent in smokers than in nonsmokers (10, 17). Yokode et al.(18) demonstrated that low density lipoprotein (LDL) pretreated with cigarette smoking was easily modified and accumulated in macrophages. An immunohistochemical study revealed that a high level of smoking was accompanied by oxidized LDL deposition in the atherosclerotic lesions (19). These reports support the evidence that cigarette smoking accelerates the progression of atherosclerosis, especially that of lipid-rich types. It is also reported that coronary atherosclerosis is enhanced by the combination of hyperlipidemia and cigarette smoking (10). Further studies of the relation between coronary atherosclerosis and smoking index will be needed in Japanese youths.

It has recently been recognized that the rupture or erosion of atherosclerotic plaques associated with obstructive thrombus formation induces acute myocardial infarction (20). About $70 \%$ of the plaques responsible for such cardiovascular events have been reported to show less than $50 \%$ stenosis, and the vast majority of the plaques are less than $70 \%$ stenosis (21-23). It has been also reported that plaque rupture was noted in $69 \%$ and plaque erosion in $31 \%$ of patients who had died suddenly of coronary artery disease, and that plaque erosion was more often observed in younger individuals and women $(24,25)$. In addition, these lesions have less luminal narrowing, less calcification, and fewer foci of macrophages compared with those of plaque rupture (25). These lines of evidence indicate that not only lipid-rich lesions but also fibrous lesions, even those with less than $50 \%$ stenosis, are predictors of acute coronary events. These lesions were observed frequently in the coronary arteries of Japanese youths in this study.

Further attention therefore should be paid to elucidate the mechanisms in the development of predominantly fibrous lesions, because these are considered to be very important for the prevention of coronary atherosclerosis and also acute coronary events in Japanese. There are several limitations of this study, especially in the number of cases examined; only one case under 10 years of age and a paucity of female cases. Additional studies are needed to confirm the results obtained in this study. 
Acknowledgements: The authors wish to thank the following pathologists for the permission to examine autopsy samples: Drs. K. Sueishi and M. Tsuneyoshi (Kyushu University), H. Hashimoto and Y. Sasaguri (University of Occupational and Environmental Health), H. Yoshinaga and K. Takahashi (Kumamoto University), H. Yoshida and E. Sato (Kagoshima University), T. Hayashi (Miyazaki Prefectural Hospital) and H. Miyayama (Kumamoto City Hospital). We also greatly thank Ms. Ritsuko Sotomura for technical assistance. This work was partly supported by a Grant-in-Aid for Encouragement of Young Scientists (10770080) from the Ministry of Education, Science, Sports and Culture, Japan, and by the National Cardiovascular Center.

\section{References}

(1) Grundy SM, Greenland P, Herd A, Huebsch JA, Jones RJ, Mitchell $\mathrm{JH}$, and Schlant RC: Cardiovascular and risk factor evaluation of healthy American adults. A statement for physicians by an Ad Hoc Committee appointed by the Steering Committee, American Heart Association. Circulation, 75: 1340A-1362A, 1987

(2) Sekimoto H, Goto Y, Goto Y, Naito C, Yasugi T, Okido M, Kuzuya F, Takeda R, Yamamoto A, Fukuzaki H, Kajiyama G, Kokubu T, Uzawa H, Mimura G, and Shimada O : Changes of serum total cholesterol and triglyceride levels in normal subjects in Japan in the past twenty years. Jpn Circul J, 47: 1351-1358, 1983

(3) PDAY Research Group: Natural history of aortic and coronary atherosclerotic lesions in youth: Findings from the PDAY study. Arterioscler Thromb, 13:1291-1298, 1993

(4) Kragel AH, Reddy SG, Wittes JT, and Roberts WC: Morphometric analysis of the composition of atherosclerotic plaques in the four major epicardial coronary arteries in acute myocardial infarction and in sudden coronary death. Circulation, 80: 1747-1756, 1989

(5) Solberg LA and Strong JP: Risk factors and atherosclerotic lesions: A review of autopsy studies. Arteriosclerosis, 3: 187-198, 1983

(6) Newman WP 3d, Wattigney W, and Berenson GS: Autopsy studies in United States children and adolescents. Relationship of risk factors to atherosclerotic lesions. Ann NY Acad Sci, 623: 16-25, 1991

(7) Tanaka K, Masuda J, Imamura T, Sueishi K, Nakashima T, Sakurai I, Shozawa T, Hosoda Y, Yoshida Y, Nishiyama $Y$, Yutani $C$, and Hatano $S$ : A nation-wide study of atherosclerosis in infants, children and young adults in Japan. Atherosclerosis, 72 : 143-156, 1988

(8) Nakamura $\mathrm{H}$ and Sakurai I: Intimal cell population and location in arteries of Japanese children and youth. Angiology, 43 : 229-243, 1992

(9) Davies MJ: The pathology of ischaemic heart disease. In: Recent Advances in Histopathology. ed by Anthony PP and MacSween PNM, pp 185-201, Churchill Livingstone, Edinburgh, 1987

(10) Wissler RW, Strong JP, and the PDAY Research Group: Risk factors and progression of atherosclerosis in youth. Am J Pathol, 153: 1023-1033, 1998
(11) Ross R: The pathogenesis of atherosclerosis -an update. N Engl J Med, 314: 488-500, 1986

(12) Steinberg $D$ : Oxidative modification of LDL and atherogenesis. Circulation, 95 : 1062-1071, 1997

(13) Komatsu A, Sakurai I, and the Pathobiological Determinants of Atherosclerosis in Youth (PDAY) Research Group: A study of the development of atherosclerosis in childhood and young adults: Risk factors and the prevention of progression in Japan and the USA. Pathol Int, 46: $541-547,1996$

(14) Sakurai I, Miyakawa K, Komatsu A, and Sawada T: Atherosclerosis in Japanese youth with reference to differences between each artery. Ann NY Aacd Sci, 598: 410-417, 1990

(15) Velican C and Velican D: Methods for evaluating the natural history of coronary atherosclerosis. In : Natural History of Coronary Atherosclerosis. ed by Velican C and Velican D, pp 67-104, CRC Press Inc, Florida, 1989

(16) Kannel WB, Castelli WP, Gordon T, and McNamara PM : Serum cholesterol, lipoproteins, and the risk of coronary heart disease. The Framingham study. Ann Intern Med, $74: 1-12,1971$

(17) Tracy RE, Toca VT, Strong JP, and Richards ML: Relationship of raised atherosclerotic lesions to fatty streaks in cigarette smokers. Atherosclerosis, 38: 347-357, 1981

(18) Yokode M, Kita T, Arai H, Kawai C, Narumiya S, and Fujiwara $M$ : Cholesteryl ester accumulation in macrophages incubated with low density lipoprotein pretreated with cigarette smoke extract. Proc Natl Acad Sci USA 85: 2344-2348, 1988

(19) Scanlon CEO, Berger B, Malcom G, Wissler RW, and PDAY Research Group: Evidence for more extensive deposits of epitopes of oxidized low density lipoprotein in aortas of young people with elevated serum thiocyanate levels. Atherosclerosis, 121: 23-33, 1996

(20) Fuster V, Badimon L, Badimon JJ, and Chesebro JH: The pathogenesis of coronary artery disease and the acute coronary syndromes. N Engl J Med, 326: 242 250, 1992

(21) Falk E, Shah PK, and Fuster V : Coronary plaque disruption. Circulation, 92: 657-671, 1995

(22) Ambrose JA, Tannenbaum MA, Alexopoulos D, Hjemdahl-Monsen CE, Leavy J, Weiss M, Borrico S, Gorlin R, and Fuster $\mathrm{V}$ : Angiographic progression of coronary artery disease and the development of myocardial infarction. J Am Coll Cardiol, 12: 56-62, 1988

(23) Little WC, Constantinescu M, Applegate RJ, Kutcher MA, Burrows MT, Kahl FR, and Santamore WP : Can coronary angiography predict the site of a subsequent myocardial infarction in patients with mild-to moderate coronary artery disease? Circulation, 78: 1157-1166, 1988

(24) Burke AP, Farb A, Malcom GT, Liang Y, Smialek J, and Virmani R: Coronary risk factors and plaque morphology in men with coronary disease who died suddenly. $\mathrm{N}$ Engl J Med, 336: 1276-1282, 1997

(25) Farb A, Burke AP, Tang AL, Liang Y, Mannan P, Smialek $\mathrm{J}$, and Virmani $\mathrm{R}$ : Coronary plaque erosion without rupture into a lipid core: A frequent cause of coronary thrombosis in sudden coronary death. Circulation, 93 : 1354-1363, 1996 